\title{
Relationship between the quantity of oral Candida and systemic condition/diseases of the host: Oral Candida increases with advancing age and anemia
}

\author{
Fumihiro Nishimaki ${ }^{1}$, Shin-ichi Yamada ${ }^{1}$, , Makiko Kawamoto ${ }^{1}$, Akinari Sakurai ${ }^{1}$, Kiyonori Hayashi ${ }^{1}$, \\ Hiroshi Kurita ${ }^{1}$ \\ ${ }^{1}$ Department of Dentistry and Oral Surgery, Shinshu University School of Medicine. Matsumoto, Japan \\ *Corresponding author: Shin-ichi Yamada, DDS, PhD \\ Department of Dentistry and Oral Surgery \\ Shinshu University School of Medicine \\ 3-1-1, Asahi, Matsumoto, 390-8621, Japan \\ Tel.: +81 (0)263-37-2675, Fax: +81 (0)263-37-2676 \\ E-mail: yshinshin@shinshu-u.ac.jp
}

Running title: Relationship between oral Candida and medical health conditions

\begin{abstract}
Background: The impact of host systemic conditions/diseases on the prosperity of oral Candida colonies remains unclear. The aim of the present study was to investigate whether a relationship exists between the quantity of oral Candida and the systemic condition/diseases of the host.

Patients and methods: The cross-sectional relationship between Candida mannan concentrations and health check-up results was analyzed in consideration of local conditions that influence the prevalence of oral Candida.

Results: Candida mannan concentrations correlated with age, the number of untreated decayed teeth, number of prosthetic teeth, salivary $\mathrm{pH}, \mathrm{HbAlc}$, and the red blood cell count in a univariate analysis. In a multivariate analysis, Candida mannan concentrations correlated with age, the number of untreated decayed teeth, number of prosthetic teeth, salivary $\mathrm{pH}$, and the red blood cell count. Candida mannan concentrations were higher in subjects older than 80 years, with a higher number of either untreated or prosthetic teeth, with a lower salivary $\mathrm{pH}$, and with a decreased red
\end{abstract}


blood cell count. Mannan concentrations were slightly higher in subjects with elevated HbA1c.

Conclusions: The present results suggest a close relationship between the quantity of oral Candida and the systemic condition/diseases of the host. Oral Candida may increase in immunocompromised hosts.

Key words: oral Candida, systemic condition, systematic disease, age, anemia

\section{Introduction}

Candida species are commensal fungal organisms as well as opportunistic pathogens of mucosal tissues. Candida habitually resides in the oral cavity and a number of systemic and local factors may cause an increase in Candida species.

Previous studies reported that the amount of oral Candida increased under specific oral conditions, including denture wearing, hyposalivation, low salivary $\mathrm{pH}$, and the presence of dental carious lesions [1-6]. However, an increase in oral Candida has also been suggested to be closely related to host immunity. While HIV-infected individuals were previously found to have a similar prevalence of oral Candida carriage to that of a control group, the immune status of the host (CD4-positive cell count) was suggested to influence the oral colonization of Candida [7]. Furthermore, the prevalence of Candida in the oral cavity was shown to be higher in transplant recipients than in immunocompetent control subjects. Kidney or liver transplantation predisposes patients to the development of an increased density of Candida colonies [8]. We previously demonstrated that the amount of oral Candida was greater in hosts with a lower immunological status [9]. Correlations have also been reported between the amount of oral Candida and the numbers of T cells, naïve T cells, and Natural Killer (NK) cells [9]. These findings suggest that oral Candida increases in immunocompromised individuals, including HIV-positive and AIDS patients, organ transplant recipients, and patients who undergo immunosuppressive and/or cytotoxic anticancer chemotherapy [10-16]. On the other hand, other systemic conditions or diseases have been suggested to impair immunity, such as aging, malnutrition, obesity, malignancy, diabetes mellitus (DM), and anemia. However, limited information is currently available on the impact of these conditions/diseases on the prosperity of oral Candida colonies. 
Although the conventional detection method for oral Candida is a culture of oral samples (i.e., smear, swab, or imprint specimen; whole saliva; and oral rinse solution), previous studies demonstrated the low positivity of cultures [17-19]. On the hand, Candida mannan antigen detection is a reliable and sensitive method for the serological diagnosis of systemic candidiasis, and an assay kit is commercially available [20-22]. The Unimedi Candida kit is a microplate enzyme-linked immunosorbent assay (ELISA) using affinity-purified polyclonal antibodies against Candida albicans mannan. Although ELISA methods has generally time-consuming nature, the Unimedi Candida test has been reported to be more sensitive and specific than other commercially available mannan antigen detection kits using a monoclonal antibody against $C$. albicans mannan [21,23]. This assay was reported to have high sensitivity for mannanemia with C. albicans, C. tropical, C. guilliermondii, and C. lusitanite and low sensitivity with C. glabrata, C. parasilosis, and C. krusei [21,23].

Therefore, the aim of the present study was to investigate whether a relationship exists between the quantity of oral Candida and systemic condition/diseases of the host. We examined the quantity of oral Candida using the Candida mannan antigen in patients who underwent specific health check-ups [24]. We also examined the cross-sectional relationship between Candida mannan concentrations and health check-up results while considering local conditions that influence the prevalence of oral Candida.

\section{Materials and methods}

This study protocol was approved by the Committees on Medical Research of Shinshu University (\#2795 and \#3683) and Aizawa Hospital (\#2012-091).

\section{Candida mannan antigen and Candida colonies in cultures}

Oral rinse solutions obtained from 32 subjects (18 patients and 14 healthy volunteers) were used in the present study. Informed consent was obtained from all subjects. They included 13 men and 19 women, with a mean age of 53.4 years (range: $26-88$ years) (Table 1). Oral rinse solution was collected by rinsing the mouth with 5 $\mathrm{mL}$ of sterile saline, which was held in the mouth for 30 seconds before collection in a sterile container. Half of the solution was used for cultivation and the remaining was submitted for the detection of the Candida mannan antigen.

Concentrated oral rinse solution was prepared by centrifuging the rinse solution at $3,200 \times g$ for 20 minutes. After the supernatant was removed, the precipitate was resuspended in $250 \mu \mathrm{L}$ saline and $50 \mu \mathrm{L}$ of the sample was inoculated onto Chromagar 
Candida agar (Kyokuto Pharmaceutical Industrial Co., Ltd., Tokyo, Japan). Candida colonies on culture agar were counted after an incubation at $37{ }^{\circ} \mathrm{C}$ for 48 hours [25].

An assay of the Candida mannan antigen was conducted at SRL Inc. (SRL. Inc., Tokyo, Japan). Briefly, the Mannan antigen was measured using a commercial sandwich ELISA, Unimedi Candida (Kyokuto Pharmaceutical Industry Co., Ltd., Tokyo, Japan). The test was performed according to the manufacturer's instructions. Briefly, oral rinse solution $(150 \mu \mathrm{L})$ was mixed with $150 \mu \mathrm{L}$ of treatment solution (including $20 \mathrm{nmol} / \mathrm{L}$ of sodium dihydrogen phosphate), boiled for 4 minutes, and then centrifuged at $10,000 \times g$ for 10 minutes. The supernatant $(100 \mu \mathrm{L})$ was added to the wells of antibody-coated microtiter plates. After an incubation at room temperature for 2 hours, the plates were washed thoroughly, and $100 \mu \mathrm{L}$ of alkaline phosphatase-conjugated antibodies was added to the wells. After a further incubation at room temperature for 1 hour, the plates were washed. The reaction was revealed by an incubation with $100 \mu \mathrm{L}$ of substrate solution at room temperature for 20 minutes. After the addition of $100 \mu \mathrm{L}$ stopping solution to the wells, optical density was read at 490-500 $\mathrm{nm}$ on a microplate reader. Each experiment included positive and negative control samples as well as a calibration curve, which was made with a pool of normal human serum supplemented with known concentrations of mannan. The relationship between mannan concentrations in the immunoassay $(\mathrm{U} / \mathrm{ml})$ and the number of Candida colonies in cultures (CFU/culture) was analyzed.

Table 1. The characteristics of individuals analyzed and the concentration of oral Candida mannan antigen.

\begin{tabular}{|c|c|c|c|}
\hline Characteristics & (n) & $\begin{array}{l}\text { Amount of Candida (CFU) } \\
\text { median (IQR) }\end{array}$ & \\
\hline \multirow[t]{2}{*}{ Sex } & Women (19) & $6 \quad(1.5-160)$ & $\mathrm{p}=0.81^{*}$ \\
\hline & Men (13) & $10 \quad(4-15)$ & \\
\hline \multirow[t]{2}{*}{ Age } & $20-59(19)$ & $4 \quad(1-6)$ & $\mathrm{p}<0.01^{*} \quad \mathrm{p}<0.001^{* *}$ \\
\hline & $60<(13)$ & $285(20-2160)$ & $(r=0.64)$ \\
\hline \multirow[t]{3}{*}{ Oral symptoms/signs } & Absence (19) & $4 \quad(1.25-6)$ & \\
\hline & BMS (6) & $10 \quad(1-162)$ & $\mathrm{p}=0.19 *($ vs. absence $)$ \\
\hline & Oral candidiasis (7) & $2320(160-2900)$ & $\mathrm{p}<0.001^{*}$ (vs. absence) \\
\hline \multirow[t]{2}{*}{ Denture wearing } & $(-)(19)$ & $4 \quad(1-6.5)$ & $\mathrm{p}<0.001^{*}$ \\
\hline & $(+)(13)$ & $285 \quad(15-2610)$ & \\
\hline \multirow[t]{2}{*}{ Systemic disease } & Absence (14) & $4 \quad(1.3-5.8)$ & $\mathrm{p}<0.01^{*}$ \\
\hline & Presence (18) & $24.5(10.3-1813.8)$ & \\
\hline
\end{tabular}




\section{Specific health check-up and dental check-up}

The present study performed specific health check-ups [24] at Azumino and Shiojiri City, Nagano Prefecture, Japan. In 2017, 1,935 persons insured by the national health insurance system and older than 30 years (including self-employed workers, farmers, and the elderly) underwent specific health and dental check-ups. Of these, 563 individuals (261 men and 302 women) were randomly selected and included in the present study. Written informed consent was obtained from all individuals.

Specific health check-ups were conducted following the standard program supplied by the Ministry of Health, Labour and Welfare of Japan (2013) [24], and included a medical interview, measurements of body height, weight, abdominal circumference, and blood pressure, and a blood examination. The blood examination measured the levels of triglycerides, low/high-density lipoprotein cholesterol, blood sugar, creatinine, and $\mathrm{HbA1c}$ as well as the red blood cell count. Each individual also underwent a dental check-up by trained seven dentists. The dental check-up included the inspection of dental and periodontal tissues and an assessment of the dryness of the mouth, according to the method of the Japanese National Survey of Dental Diseases. All examinations were conducted using a plane dental mirror and explorer using sunlight or a flashlight. However, the explorer was only used to clean the tooth surface as necessary and not to probe teeth or tooth surfaces. In order to evaluate the prevalence of dental diseases, DMFT indices were recorded. Since 1938, DMF indices became a relevant tool for monitoring the distribution of dental caries; it was applied by the World Health Organization (WHO) in their assessment of oral health, reflecting the intensity or frequency of dental caries [26]. Additionally, the use of dentures was verified. Regarding the dentist's calibration of measuring of pocket depth, and evaluating of dental caries and oral hygiene, all dentists were trained with models. The dryness of the mouth was judged by the dentist and classified into four categories according to the clinical classification reported by Kakinoki (normal: non-dry, slight: saliva shows viscosity, moderate: saliva shows tiny bubbles on the tongue, and severe: dry tongue with little or no saliva present) [27]. Prior to the dental examination, oral rinse solution was collected by rinsing the mouth for 10 seconds with $3 \mathrm{ml}$ of distilled water. Salivary $\mathrm{pH}$ was measured using the salivary multi-test system (Salivary Multi Test, Lion Co., Tokyo, Japan). Approximately $1 \mathrm{ml}$ of the oral rinse solution was submitted to the measurement of the Candida mannan antigen. The assay of the Candida mannan antigen was described above.

The relationships between mannan concentrations and factors influencing the 
prosperity (quantity) of oral Candida, including sex, age, dental caries, prosthetic teeth, the dryness of the mouth, salivary $\mathrm{pH}$, obesity (BMI: body mass index), DM (HbAlc), renal function (estimate glomerular filtration rate: eGFR), and anemia (red blood cell count), were then analyzed.

Statistical analyses were performed using JMP ver.13 (SAS Institute Inc., North Carolina, USA). The relationship between mannan concentrations in the immunoassay $(\mathrm{U} / \mathrm{ml})$ and the number of Candida colonies in cultures (CFU/culture) was examined with Pearson's correlation coefficient. A univariate analysis was performed with the Wilcoxon rank-sum test and Spearman's rank correlation. A multivariate analysis was conducted with a stepwise multiple regression analysis. P-values $<0.05$ were considered to be significant.

Fig. 1 Relationship between the Candida mannan antigen and Candida colonies in cultures. A strong correlation was observed (Pearson's correlation coefficient, $r=0.74$, $\mathrm{p}<0.01)$.

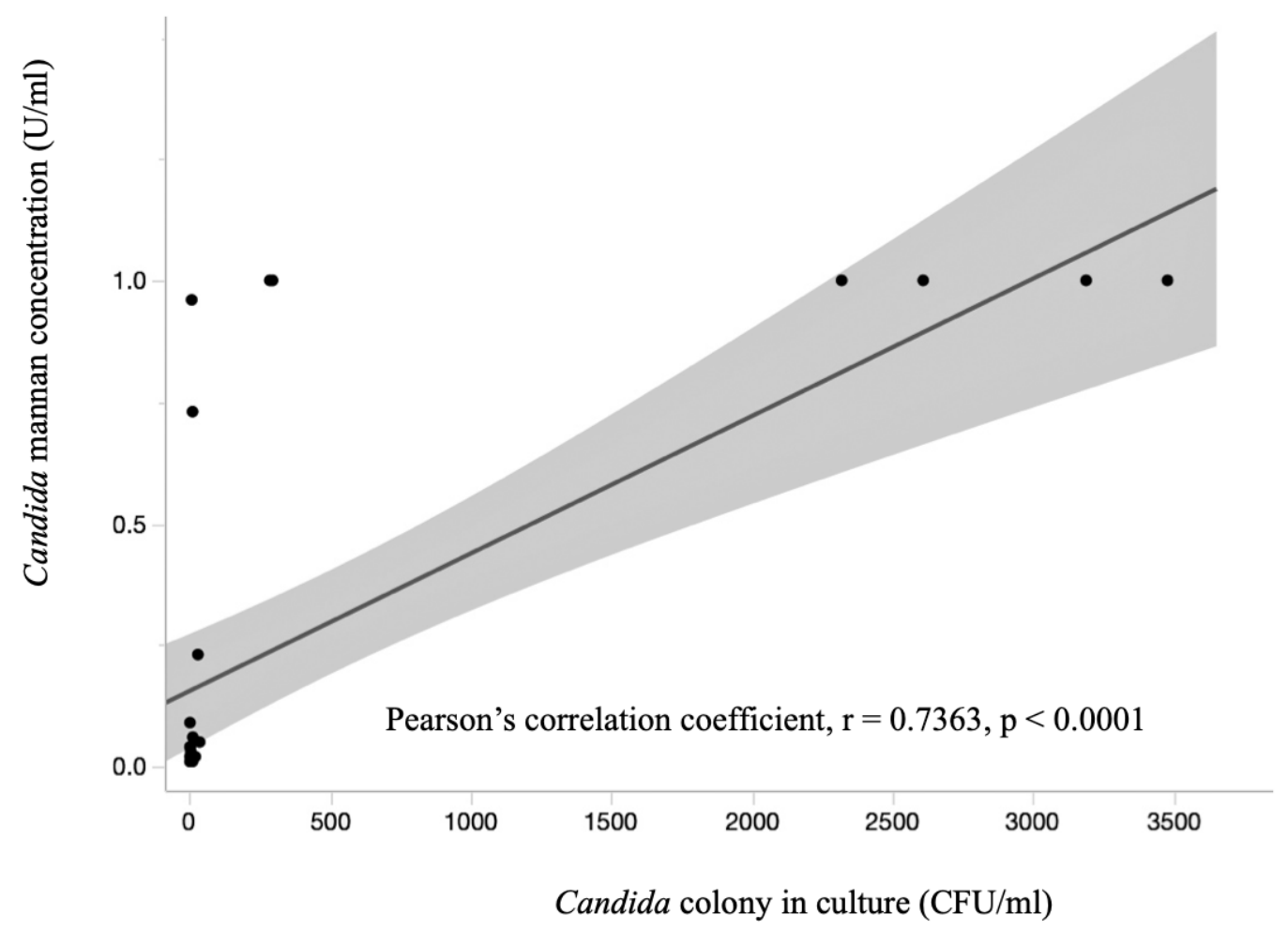




\section{Results}

\section{Relationship between the Candida mannan antigen and Candida colonies in cultures}

Oral Candida was detected in the cultures of samples from all subjects. The median number of Candida colonies was $6.5 \mathrm{CFU} / \mathrm{ml}$ (interquartile range (IQR): $28.5 \mathrm{CFU} / \mathrm{ml}$, range: $1-3,480 \mathrm{CFU} / \mathrm{ml})$. Candida mannan was also detected in all samples. The median concentration was $0.025 \mathrm{U} / \mathrm{ml}$ (IQR: $0.89 \mathrm{U} / \mathrm{ml}$ ) with a range between 0.01 and 1.00. The relationship between the Candida culture and mannan antigen concentrations is shown in Figure 1, with a strong correlation being observed (Pearson's correlation coefficient, $\mathrm{r}=0.74, \mathrm{p}<0.01$ ).

The results of univariate analyses on the relationship between the quantity of oral Candida colonies and oral and systemic health conditions were also shown in Table 1. Oral Candida was more prevalent in elderly subjects (older than 60 years), denture wearers, and those with systemic diseases $(\mathrm{p}<0.05)$.

\section{Relationship between the quantity of oral Candida and oral and systemic health conditions}

A total of 563 out of 1,935 individuals were randomly selected and included in the present study. Their characteristics and the results of dental and health check-ups were summarized in Table 2.

The results of univariate analyses on the relationship between the quantity of oral Candida and oral and systemic health conditions were also shown in Table 2. Candida mannan concentrations correlated with age $(\mathrm{p}<0.01)$, the number of untreated decayed teeth $(\mathrm{p}<0.01)$, the number of prosthetic teeth $(\mathrm{p}<0.01)$, salivary $\mathrm{pH}(\mathrm{p}<0.01)$, HbA1c $(p<0.05)$, and the red blood cell count $(p<0.01)$. Mannan concentrations increased with advancing age, and were high in those older than 60 years. The greater the number of decayed or prosthetic teeth, the higher the mannan concentration. The lower the salivary $\mathrm{pH}$, the higher the Candida mannan concentration. Mannan concentrations were higher in subjects with elevated HbAlc. They were also higher in subjects with advancing anemia. Furthermore, mannan concentrations were slightly high in subjects with moderate to severe renal dysfunction (eGFR less than $45, \mathrm{p}=$ 0.073). Mannan concentrations were also slightly higher in subjects with moderate dry mouth than in those with no or slightly dry mouth.

The results of the multivariate analysis were shown in Table 3. The multivariate analysis was included the significant variables in the univariate analysis and variable, which was detected the association with Candida concentration. The age decade, 
number of decayed teeth, number of prosthetic teeth, dryness of the mouth, salivary $\mathrm{pH}$, $\mathrm{HbA} 1 \mathrm{C}$, and the red blood cell count were included in the model. eGFR was excluded from the model because of its correlation with other independent variables (a correlation was observed between eGFR and salivary $\mathrm{pH}, \mathrm{r}=0.025, \mathrm{p}<0.001$ ).

Candida mannan concentrations correlated with age $(\mathrm{p}<0.01)$, the number of untreated decayed teeth $(\mathrm{p}<0.01)$, number of prosthetic teeth $(\mathrm{p}<0.01)$, salivary $\mathrm{pH}(\mathrm{p}$ $<0.01)$, and the red blood cell count $(\mathrm{p}<0.01)$. Mannan concentrations were higher in subjects older than 80 years. Candida mannan concentrations increased in subjects with a higher number of either untreated or prosthetic teeth. They were also higher in subjects with lower salivary $\mathrm{pH}$ and red blood cell counts. HbA1c was associated with the quantity of oral Candida $(\mathrm{p}=0.078)$.

Table 2. Relationship between oral Candida mannan concentrations and results of dental and health examinations (univariate analysis).

\begin{tabular}{|c|c|c|c|c|c|c|c|}
\hline Variables & & $\mathbf{n}$ & Average & SE & Median & IQR & \\
\hline \multirow[t]{2}{*}{ Sex } & Woman & 302 & 0.22 & 0.02 & 0.02 & $0.02-0.26$ & NST \\
\hline & Man & 261 & 0.19 & 0.02 & 0.02 & $0.02-0.19$ & \\
\hline \multirow{6}{*}{ Age decade (years) } & $30-39$ & 19 & 0.08 & 0.07 & 0.03 & $0.02-0.1$ & $p<0.01 *$ \\
\hline & $40-49$ & 55 & 0.09 & 0.04 & 0.02 & $0.02-0.03$ & \\
\hline & $50-59$ & 55 & 0.11 & 0.04 & 0.02 & $0.02-0.05$ & \\
\hline & $60-69$ & 183 & 0.17 & 0.02 & 0.02 & $0.02-0.19$ & \\
\hline & $70-79$ & 195 & 0.23 & 0.02 & 0.04 & $0.02-0.33$ & \\
\hline & $80 \leq$ & 56 & 0.46 & 0.04 & 0.31 & $0.02-1$ & \\
\hline \multirow[t]{6}{*}{ Number of untreated decayed teeth } & & 402 & 0.18 & 0.01 & 0.02 & $0.02-0.2$ & $p<0.01^{*}$ \\
\hline & 1 & 100 & 0.19 & 0.03 & 0.02 & $0.02-0.14$ & \\
\hline & 2 & 27 & 0.27 & 0.07 & 0.05 & $0.02-0.47$ & \\
\hline & 3 & 14 & 0.51 & 0.11 & 0.34 & $0.12-1$ & \\
\hline & 4 & 11 & 0.50 & 0.14 & 0.27 & $0.04-1$ & \\
\hline & $5 \leq$ & 9 & 0.30 & 0.11 & 0.11 & $0.03-0.54$ & \\
\hline \multirow[t]{5}{*}{ Number of prosthetic teeth } & 0 & 273 & 0.11 & 0.02 & 0.02 & $0.02-0.08$ & $p<0.01 *$ \\
\hline & 1 & 86 & 0.16 & 0.03 & 0.02 & $0.02-0.175$ & \\
\hline & 2 & 56 & 0.26 & 0.04 & 0.04 & $0.02-0.41$ & \\
\hline & 3 & 23 & 0.28 & 0.06 & 0.13 & $0.02-0.39$ & \\
\hline & $4 \leq$ & 125 & 0.40 & 0.03 & 0.19 & $0.02-0.93$ & \\
\hline \multirow[t]{3}{*}{ Dryness of mouth } & Normal & 525 & 0.20 & 0.01 & 0.02 & $0.02-0.22$ & $N S *$ \\
\hline & Slight & 26 & 0.25 & 0.07 & 0.02 & $0.02-0.38$ & \\
\hline & Moderate & 5 & 0.58 & 0.20 & 0.64 & $0.12-1$ & \\
\hline \multirow[t]{3}{*}{ pH of saliva } & $7.04<$ & 78 & 0.13 & 0.03 & 0.02 & $0.02-0.07$ & $p<0.01^{*}$ \\
\hline & $6.7-7.04$ & 115 & 0.17 & 0.03 & 0.02 & $0.02-0.19$ & \\
\hline & $<6.7$ & 365 & 0.23 & 0.02 & 0.04 & $0.02-0.32$ & \\
\hline \multirow[t]{3}{*}{ BMI } & $<18.5$ & 50 & 0.20 & 0.04 & 0.02 & $0.02-0.27$ & $p<N S^{*}$ \\
\hline & $18.5-24.9$ & 381 & 0.21 & 0.02 & 0.02 & $0.02-0.21$ & \\
\hline & $25.0 \leq$ & 131 & 0.21 & 0.03 & 0.03 & $0.02-0.31$ & \\
\hline \multirow[t]{3}{*}{ HbA1c (NGSP) } & $\leq 5.5$ & 197 & 0.17 & 0.02 & 0.02 & $0.02-0.15$ & $p<0.05^{*}$ \\
\hline & $5.6-6.4$ & 314 & 0.22 & 0.02 & 0.02 & $0.02-0.3$ & \\
\hline & $5.6 \leq$ & 51 & 0.26 & 0.05 & 0.07 & $0.02-0.35$ & \\
\hline \multirow[t]{3}{*}{ e-GFR } & $\geq 60$ & 471 & 0.19 & 0.01 & 0.02 & $0.02-0.21$ & $p=0.073^{*}$ \\
\hline & $45-59$ & 74 & 0.29 & 0.05 & 0.025 & $0.02-0.55$ & \\
\hline & $<45$ & 16 & 0.31 & 0.10 & 0.06 & $0.02-0.52$ & \\
\hline \multirow[t]{3}{*}{ Red blood cell count } & $\mathrm{M}: \geq 400, \mathrm{~F}: \geq 360$ & 544 & 0.20 & 0.01 & 0.02 & $0.02-0.22$ & $p<0.01^{*}$ \\
\hline & M: $360-399, F: 330-359$ & 15 & 0.36 & 0.09 & 0.23 & $0.02-0.55$ & \\
\hline & $\mathrm{M}:<360, \mathrm{~F}:<330$ & 3 & 0.53 & 0.22 & 0.73 & $0.09-0.78$ & \\
\hline
\end{tabular}

$\dagger:$ Wilcoxon rank-sum test

*: Spearman's rank correlation 
Table 3. Relationship between oral Candida mannan concentrations and results of dental and health examinations (multivariate analysis).

\begin{tabular}{|c|c|c|c|c|c|c|}
\hline & $\begin{array}{l}\text { Partial regression } \\
\text { coefficient }\end{array}$ & Standard error & $\begin{array}{l}\text { standard partial } \\
\text { regression coefficient }\end{array}$ & t-value & p-value & $\begin{array}{l}\text { Variance Inflation } \\
\text { Factor }\end{array}$ \\
\hline 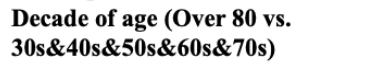 & 0.087 & 0.021 & 0.166 & 4.26 & $<.0001^{*}$ & 1.106 \\
\hline Number of untreated decade teeth & 0.027 & 0.009 & 0.116 & 3.12 & $0.0019 *$ & 1.009 \\
\hline Number of prosthetic teeth & 0.017 & 0.002 & 0.304 & 7.88 & $<.0001^{*}$ & 1.083 \\
\hline Dryness of mouth & 0.056 & 0.042 & 0.050 & 1.34 & 0.1821 & 1.015 \\
\hline Red Blood Cell count & -0.001 & 0.000 & -0.152 & -4.00 & $<.0001^{*}$ & 1.047 \\
\hline
\end{tabular}

\section{Discussion}

Candida species are frequently isolated from the oral cavity of healthy individuals of all ages, and have been reported at a prevalence of $15-75 \%$ [28-30]. The amount of oral Candida has been shown to increase under specific oral conditions, including denture wearing, hyposalivation, low salivary $\mathrm{pH}$, and the presence of dental carious lesions [1-6]. Although other systemic conditions or diseases impair immunity, such as aging, malnutrition, obesity, malignancy, DM, and anemia, the impact of these conditions/diseases on the prosperity of oral Candida colonies remains unclear. Therefore, the aim of the present study was to investigate the relationship between the quantity of oral Candida and the systemic condition/diseases of the host.

The Unimedi Candida kit is was reported to have high sensitivity for mannanemia with C. albicans [21,23]. Furthermore, we previously demonstrated that Candida mannan antigen detection has potential as a sensitive technique for the detection of oral Candida, and also that the conditions of the ELISA-based assay need to be optimized for oral rinse solutions [31]. Candida mannan was detected in all samples in the present study, and a correlation was observed between Candida cultures and mannan antigen concentrations. Therefore, the quantity of oral Candida using the Candida mannan antigen in subjects who underwent specific health check-ups was examined in the present study.

The multivariate analysis revealed that Candida mannan concentrations correlated with some intra-oral conditions, including decayed teeth, prosthetic teeth, and salivary $\mathrm{pH}$. These results were consistent with previous findings. A correlation was reported between the number of caries teeth and Candida colony-forming units in saliva [32]. The enzymes secreted by $C$. albicans degrade collagen, particularly type I, which represents more than $90 \%$ of the dentin organic matrix [33-39]. C. albicans colonization and proliferation is facilitated, which may contribute to the promotion of the carious process in dentin [40-42]. The elimination of dentinal caries may reduce the number of 
sites for Candida colonization and, consequently, the risk of fungal infections [43]. Oral Candida colonization was previously reported to increase by six-fold in denture wearers $[44,45]$. A spongy denture tissue surface, the surface characteristics of denture base acrylic resins, such as hydrophobicity, and an abundance of nutritive substances are considered to be an ideal incubator for species such as C. albicans [46,47]. A previous study demonstrated that salivary $\mathrm{pH}$ was lower in complete denture wearers than in non-denture wearers [48], and low $\mathrm{pH}$ levels promoted the adhesion and proliferation of Candida yeast. Decreased salivary gland hypofunction and $\mathrm{pH}$ changes have been noted in the elderly, the main wearer of dentures [48]. Furthermore, a reduction in the salivary flow rate with aging induced an increase in the concentration of microbes in saliva [48]. Since these factors support the present results, a strong correlation may exist between the number of untreated decayed teeth, number of prosthetic teeth, salivary $\mathrm{pH}$, and the prevalence of oral Candida. However, in recent preliminary genetic study of the oral mycobiome of children with and without dental caries, C. albicans was reported to be frequently isolated from both caries-affected and caries-free dentition, and not to be more abundant in children with dental caries [49]. The further investigation will be needed to clarify the association between dental caries and C. albicans.

On the other hand, the results of the multivariate analysis revealed that some systemic factors also had a significant impact on the Candida load in the oral cavity. Besides the local factors described above, age and the red blood cell count independently influenced oral Candida mannan concentrations. Furthermore, HbA1c influenced the quantity of oral Candida. Aging is one of the important factors for oral Candida infection. The elderly have been reported to have several metabolic disorders including decreased hepatic and renal functions [50], the use of multiple drugs, and poor nutrition [51], which are considered to contribute to colonization and infection by Candida [52]. A previous study investigated the relationship between Candida carriage and drugs, and a multivariate analysis revealed that the variables of an older age ( 80 years and older) had a significant impact on the quantity of oral Candida besides the presence of dry mouth [53]. These findings are consistent with the present results. The immune system becomes weaker with aging, and this decline with age is reflected in increased susceptibility to infectious diseases, poorer responses to vaccinations, and the increased prevalence of cancer and autoimmune and other chronic diseases. Innate and adaptive immune responses are affected by the aging process [54]. Aging of the immune system may contribute to the increased load of oral Candida.

A high prevalence of oral Candida infection has been reported in patients with iron deficiency anemia [55]. A high incidence of Candida infection of $85 \%$ was found 
in iron-deficient patients. Iron deficiency is the most common nutritional deficiency and causes half of all anemia cases worldwide [55]. The possibility of Candida infection in iron deficiency was reportedly due to impaired cellular immunity [56,57]. Iron is essential for the growth of all cells. Hassan et al. reported that humoral immunity, non-specific immunity (phagocytic activity and oxidative burst), and IL-6 levels were affected in patients with iron deficiency anemia [58]. Ekiz $\mathrm{C}$ et al. also showed that humoral, cell-mediated, and non-specific immunities and the activity of cytokines, which play an important role in various steps of immunogenic mechanisms, were influenced by iron deficiency anemia [59]. Other types of anemia, such as aplastic anemia and myelodysplastic syndromes, are conditions in which impaired immunity is induced. These results suggest that subjects with anemia have impaired immunity and, consequently, an increased load of oral Candida.

In the present study, $\mathrm{HbA} 1 \mathrm{C}$ affected the quantity of oral Candida. Patients with type $2 \mathrm{DM}$ are known to be at an increased risk of opportunistic infections, including oral candidiasis [60]. Due to elevated serum glucose levels and a weakened cellular immune system, patients with DM are susceptible to opportunistic infections. A previous study also reported that the oral carriage of Candida species was significantly higher in patients with DM than in controls [61]. The present results appear to support the effects of DM on the prevalence of oral Candida.

The strength of the present study was that it was an investigation based on a relatively large number of individuals who participated in specific health check-ups. Its limitation was that since this was a cross-sectional study, other confounding effects, such as medications, were unclear. Another limitation of this study was that dentist's calibrations of DMFT indea and oral hygine measurements might affect the outcomes of this study. For the evaluation and standardization of examiner's skill, all dentists participated in this study were trained with dental models for dentist's calibration.

In conclusion, the cross-sectional relationship between Candida mannan concentrations and health check-up results was analyzed and local conditions that influence the prevalence of oral Candida were considered. A strong correlation was observed between Candida cultures and mannan antigen concentrations. Candida mannan concentrations correlated with age, the number of untreated decayed teeth, number of prosthetic teeth, salivary $\mathrm{pH}, \mathrm{HbA} 1 \mathrm{C}$, and the red blood cell count in the univariate analysis. In the multivariate analysis, Candida mannan concentrations correlated with age, the number of untreated decayed teeth, number of prosthetic teeth, salivary $\mathrm{pH}$, and the red blood cell count. Mannan concentrations were higher in subjects older than 80 years, with a higher number of either untreated or prosthetic teeth, 
lower salivary $\mathrm{pH}$, and a low red blood cell count. The relationship between the quantity of oral Candida and systemic condition/diseases of the host was demonstrated in the present study.

Compliance with Ethical Standards

Conflict of interest The authors declare that they have no conflict of interest. The authors alone are responsible for the content and the writing of the paper.

Ethical Approval: This study protocol was approved by the Committees on Medical Research of Shinshu University (\#2795 and \#3683) and Aizawa Hospital (\#2012-091).

References

1. Arendorf TM, Walker DM. The prevalence and intra-oral distribution of Candida albicans in man. Arch Oral Biol 1980;25(1):1-10.

2. Krogh P, Hald B, Holmstrup P. Possible mycological etiology of oral mucosal cancer: catalytic potential of infecting Candida albicans and other yeasts in production of N-nitrosobenzylmethylamine. Carcinogenesis 1987 ;8(10):1543-8.

3. Samaranayake LP . Oral mycoses in HIV infection. Oral Surg Oral Med Oral Pathol $1992 ; 73(2): 171-80$.

4. Tillonen J, Homann N, Rautio M, Jousimies-Somer H, Salaspuro M. Role of yeasts in the salivary acetaldehyde production from ethanol among risk groups for ethanol-associated oral cavity cancer. Alcohol Clin Exp Res 1999 ;23(8):1409-15. 5. Correa P, Houghton J. Carcinogenesis of Helicobacter pylori. Gastroenterology 2007 ;133(2):659-72.

6. Signoretto C, Burlacchini G, Faccioni F, Zanderigo M, Bozzola N, Canepari P. Support for the role of Candida spp. in extensive caries lesions of children. New Microbiol 2009;32(1):101-7.

7. Thanyasrisung $\mathrm{P}$, Kesakomol P, Pipattanagovit P, Youngnak-Piboonratanakit $\mathrm{P}$, Pitiphat W, Matangkasombut O. Oral Candida carriage and immune status in Thai human immunodeficiency virus-infected individuals. J Med Microbiol 2014 ;63(Pt 5):753-9.

8. Olczak-Kowalczyk D, Pawłowska J, Garczewska B, Smirska E, Grenda R, Syczewska M, et al. Oral candidiasis in immunosuppressed children and young adults after liver or kidney transplantation. Pediatr Dent 2010 ;32(3):189-94.

9.Hayashi K. Tooyama H, Tanaka H, Aizawa H, Shimane T, Kurashina K, et al. Relationship between the quantity of oral Candida and immunological viror. J Oral Maxillofac Surg Med Pathol 2017;29(1):65-70. 
10. Phelan JA, Saltzman BR, Friedland GH, Klein RS. Oral findings in patients with acquired immunodeficiency syndrome. Oral Surg Oral Med Oral Pathol 1987;64(1):50-6.

11. Korting HC, Ollert M, Georgii A, Fröschl M. In vitro susceptibilities and biotypes of Candida albicans isolates from the oral cavities of patients infected with human immunodeficiency virus. J Clin Microbiol 1988;26(12):2626-31.

12. Fridkin SK, Jarvis WR. Epidemiology of nosocomial fungal infections.

Clin Microbiol Rev 1996;9(4):499-511.

13. Palmer GD, Robinson PG, Challacombe SJ, Birnbaum W, Croser D, Erridge PL, et al. Aetiological factors for oral manifestations of HIV. Oral Dis 1996;2(3):193-7.

14. Saunus JM, Kazoullis A, Farah CS. Cellular and molecular mechanisms of resistance to oral Candida albicans infections. Front Biosci 2008;13:5345-58.

15. Dineshshankar J, Sivakumar M, Karthikeyan M, Udayakumar P, Shanmugam KT, Kesavan G. Immunology of oral candidiasis. J Pharm Bioallied Sci. 2014;6(Suppl 1):S9-S12.

16. Moyes DL, Naglik JR. Mucosal immunity and Candida albicans infection. Clin Dev Immunol. 2011:346307. doi: 10.1155/2011/346307.

17. White PL, Williams DW, Kuriyama T, Samad SA, Lewis MAO, Barnes RA. Detection of Candida in concentrated oral rinse cultures by real-time PCR. J Clin Microbiol 2004;42:2101-7.

18. Ahmad S, Khan Z, Mustafa AS, Khan ZU. Semi-nested PCR for diagnosis of candidemia: comparison with culture, antigen detection, and biochemical methods for species identification. J Clin Microbiol 2002;40(7):2483-9.

19. Liguori G, Lucariella A, Colella G, De Luca A, Marinelli P. Rapid identification of Candida species in oral rinse solutions by PCR. J Clin Pathol 2007;60(9):1035-9. 20. Alam FF, Mustafa AS, Khan ZU. Comparative evaluation of (1,3)--D-glucan, mannan and antimannan antibodies, and Candida species-specific snPCR in patients with candidemia. BMC Infect Dis 2007;7:103.

21. Fujita S, Takamura T, Nagahara M, Hashimoto T. Evaluation of a newly developed down-flow immunoassay for detection of serum mannan antigens in patients with candidaemia. J Med Microbiol 2006;55(Pt 5):537-43.

22. Persat F, Topenot R, Piens MA, Thiebaut A, Dannaoui E, Picot S. Evaluation of different commercial ELISA methods for the serodiagnosis of systemic candidosis. Mycoses 2002;45(11-12):455-60. 
23. Yoshida K, Niki Y, Miyashita N, Matsushima T. Clinical utility of serum Candida mannan antigen detection kit using ELISA. Kansenshogakuzashi 2002;76(7):536-41. Japanese; English abstract.

24. Ministry of Health, Labour and Welfare. Specific Health Checkups and Specific Health Guidance. https://www.mhlw.go.jp/english/wp/wp-hw3/d1/2-007.pdf. Accessed 25 September 2018.

25. Tooyama H, Matsumoto T, Hayashi K, Kurashina K, Kurita H, Uchida M, et al. Candida concentrations determined following concentrated oral rinse culture reflect clinical oral signs. BMC Oral Health 2015;15:150. doi: 10.1186/s12903-015-0138-z. 26.Klein H, Palmer C. Studies on dental caries vs. familial resemblance in the caries experience of siblings. Public Health Rep. 1938;53:1353-64.

27.Kakinoki Y, Maki Y, Ogasawara T, Koseki T, Nishihara T, Kikutani T, et al. Guideline for Diagnosis of Dry Mouth in the Disabled. Journal of the Japanese Association for Dental Science 2008;27:30-34.(In Japanese)

28. Ghannoum MA, Jurevic RJ, Mukherjee PK, Cui F, Sikaroodi M, Naqvi A, et al. Characterization of the oral fungal microbiome (mycobiome) in healthy individuals. PLoS Pathog 2010;6(1):e1000713. doi: 10.1371/journal.ppat.1000713.

29. Zegarelli DJ. Fungal infections of the oral cavity. Otolaryngol Clin North Am 1993;26(6):1069-89.

30. Sedgley CM, Samaranayake LP. The oral prevalence of aerobic and facultatively anaerobic gram-negative rods and yeasts in Hong Kong Chinese. Arch Oral Biol 1994;39(6):459-66.

31.Kurita H, Kamata T, Zhao C, Narikawa JN, Koike T, Kurashina K. Usefulness of a commercial enzyme-linked immunosorbent assay kit for Candida mannan antigen for detecting Candida in oral rinse solutions. Oral Surg Oral Med Oral Pathol Oral Radiol Endod 2009;107(4):531-4.

32. Cerqueira DF, Portela MB, Pomarico L, Soares RMA, Souza IPR, Castro GF. Examining dentinal carious lesions as a predisposing factor for the oral prevalence of Candida spp in HIV-infected children. J Dent Child (Chic) 2007;74(2):98-103.

33. Madigan A, Murray PA, Houpt M, Catalanotto F, Feuerman M. Caries experience and cariogenic markers in HIV-positive children and their siblings. Pediatr Dent 1996;18(2):129-36.

34. Schaller M, Borelli C, Korting HC, Hube B. Hydrolytic enzymes as virulence factors of Candida albicans. Mycoses 2005;48(6):365-77. 
35. Rodier M-H, Moudni BE, Kauffmann-Lacroix C, Daniault G, Jacquemin J-L. A Candida albicans metallopeptidase degrades constitutive proteins of extracellular matrix. FEMS Microbiol Lett 1999;177(2):205-10.

36. Imbert C, Kauffmann-Lacroix C, Daniault G, Jacquemin J-L, Rodier MH. Effect of matrix metalloprotease inhibitors on the $95 \mathrm{kDa}$ metallopeptidase of Candida albicans. J Antimicrobiol Chem 2002;49(6):1007-10.

37. Kaminishi H, Hagihara Y, Tanaka M, Cho T. Degradation of bovine Achilles tendon collagen by Candida albicans proteinase. J Med Vet Mycol 1988;26(5):315-8. 38. Berkovitz BKB, Holland GR, Moxhan BJ. Dentina. In: Berkovitz, BKB, Holland GR, Moxhan BJ. Anatomia, embriologia e histologia bucal. Porto Alegre: Artmed; 2004. pp. 125-48.

39. Piesco NP. Histologia da dentina. In: Avery JK. Desenvolvimentoe histologia bucal. Porto Alegre/São Paulo: Artmed/Editora Santos; 2005. pp. 188-205.

40. Naglik JR, Challacombe SJ, Hube B. Candida albicans secreted aspartyl proteases in virulence and pathogenesis. Microbiol Mol Biol Ver 2003;67(3):400-28.

41. Manfredi M, McCullough MJ, Al-Karaawi ZM, Vescovi P, Porter SR. In vitro evaluation of virulence attributes of Candida spp., isolated from patients affected by diabetes mellitus. Oral Microbiol Immunol 2006;21(3):183-9.

42. Mardegan RC, Foglio MA, Gonçalves RB, Höfling JF. Candida albicans proteinases. Braz J Oral Sci 2006;5:944-52.

43. das Chagas MS, Portela MB, Cerqueira DF, de Souza IP, Soares RM, Castro GF. Reduction of Candida species colonization in the oral cavity of children infected with human immunodeficiency virus after dental treatment. Oral Surg Oral Med Oral Pathol Oral Radiol Endod 2009;108(3):383-8.

44. Javed F, Klingspor L, Sundin U, Altamash M, Klinge B, Engstrom PE. Periodontal conditions, oral Candida albicans and salivary proteins in type 2 diabetic subjects with emphasis on gender. BMC Oral Health 2009;9:12.

45. Shilpa Dandekeri, KrishnaPrasad D, Manoj Shetty, Chethan Hegde, Sowmya Mangalore Kumar, Maithri Jagadeesh Kakunje. Occurrence of streptococcus and Candida species and salivary $\mathrm{pH}$ in patients wearing complete denture. Int J Health Rehabil Sci 2013;2(4):198-203.

46. Acosta-Torres LS, Lopez-Marin LM, Nunez-Anita RE, Her- nandez-Padron G, Castano VM. Biocompatible metal-oxide nanoparticles: nanotechnology improvement of conventional prosthetic acrylic resins. J Nanomaterials 2011; article ID 941561, 8 pages. 
47. Gleiznys A, Zdanavičienė E, Žilinskas J. Candida albicans importance to denture wearers. A literature review. Stomatologija 2015;17(2):54-66.

48. Mima EG, Pavarina AC, Silva MM, Ribeiro DG, Vergani CE, Kurachi C, Bagnato VS. Denture stomatitis treated with photodynamic therapy: five cases. Oral Surg Oral Med Oral Pathol Oral Radiol Endod 2011;112(5):602-8.

49. Jacquelyn M. Fechney, Gina V. Browne, Neeta Prabhu, Laszlo Irinyi, et al. Preliminary study of the oral mycobiome of children with and without dental caries. J Oral MicroBiol 2019 11:1, 1536182, DOI: 10.1080/20002297.2018.1536182 50. Flevari A, Theodorakopoulou M, Velegraki A, Armaganidis A, Dimopoulos G. Treatment of invasive candidiasis in the elderly: a review. Clin Interv Aging 2013;8:1199-208.

51. Poisson P, Laffond T, Campos S, Dupuis V, Bourdel-Marchasson I. Relationships between oral health, dysphagia and undernutrition in hospitalised elderly patients. Gerodontology 2016;33(2):161-8.

52. Tosello A, Chevaux JM, Montal S, Foti B. Assessment of oral status and oro-pharyngeal candidosis in elderly in short-term hospital care. Odontostomatol Trop 2008;31(121):43-8.

53. Yamanaka K, Nakagaki H, Morita I, Maeda N, Ohara H, Tomatsu S, et al. Relationship between oral Candida species and risk factors with reference to drugs with xerostomic side effects among institutionalised elderly in Aichi Pref., Japan. Community Dent Health 2005;22(1):19-24.

54. Castelo-Branco C, Soveral I. The immune system and aging: a review. Gynecol Endocrinol 2014;30(1):16-22.

55. Lu SY. Perception of iron deficiency from oral mucosa alterations that show a high prevalence of Candida infection. J Formos Med Assoc 2016;115(8):619-27.

56. Kumar V, Choudhry VP. Iron deficiency and infection. Indian J of Pediatr 2010;77:789e93.

57. Naderi N, Etaati Z, Joibari MR, Sobhani SA, Tashnizi SH. Immune deviation in recurrent vulvovaginal candidiasis: correlation with iron deficiency anemia. Iran J Immunol 2013;10(2):118-26.

58. Hassan TH, Badr MA, Karam NA, Zkaria M, et al. Impact of iron deficiency anemia on the function of the immune system in children. Medicine (Baltimore) 2016;95(47):e5395.

59. Ekiz C, Agaoglu L, Karakas Z, Gurel N, Yalcin I. The effect of iron deficiency anemia on the function of the immune system. Hematol J 2005;5(7):579-83. 
60. Ship JA. Diabetes and oral health: an overview. J Am Dent Assoc 2003;134 Spec No:4S-10S.

61. Zomorodian K, Kavoosi F, Pishdad GR, Mehriare P, Ebrahimi H, et al. Prevalence of oral Candida colonization in patients with diabetes mellitus. J Mycol Med. 2016;26(2):103-110. 